\title{
Evidence for differential acquired drug resistance to anti-tumour necrosis factor agents in rheumatoid arthritis
}

\author{
A Finckh, J F Simard, C Gabay, P-A Guerne, for the SCQM physicians
}

Ann Rheum Dis 2006;65:746-752. doi: 10.1136/ard.2005.045062

See end of article for authors' affiliations

Correspondence to: Dr A Finckh, Division of Rheumatology, University Hospital of Geneva, Av. Beau-Séjour 26, 1211

Geneva 14, Switzerland; afinckh@post.harvard.edu

Accepted

27 November 2005

Published Online First

8 December 2005
Background: Acquired drug resistance or gradual drug failure has been described with most disease modifying antirheumatic drugs (DMARDs) and is also starting to be recognised with anti-tumour necrosis factor (anti-TNF) agents.

Objective: To study acquired drug resistance to anti-TNF agents in rheumatoid arthritis (RA).

Methods: Swiss health authorities requested continuous monitoring of patients receiving biological agents. Intensification of co-therapy with traditional DMARDs, gradual dose escalation, and drug discontinuation rates in all patients receiving infliximab, etanercept, or adalimumab, adjusting for potential confounders, were analysed. Intensification of DMARD co-therapy and time to discontinuation of the three anti-TNF agents were analysed using a proportional hazards models. Dose escalation and evolution of RA disease activity (DAS28) were analysed using a longitudinal regression model.

Results: 1198 patients contributing 1450 patient-years of anti-TNF treatment met the inclusion criteria. The rate of intensification of traditional DMARD co-therapy over time was significantly higher with infliximab (hazards ratio $=1.73$ (99\% confidence interval $(\mathrm{Cl}) 1.19$ to 2.51$)$ ) than with the two other agents. Infliximab also showed significant dose escalation over time, with an average dose increase of $+12 \% 199 \%$ $\mathrm{Cl} 8 \%$ to $16 \%$ ) after 1 year, and $+18 \%(99 \% \mathrm{Cl} 11 \%$ to $25 \%)$ after 2 years. No significant differences in discontinuation rates were seen between the three anti-TNF agents (ANOVA, $p=0.67$ ). Evolution of disease activity over time indicated a lower therapeutic response to infliximab (DAS28, $p<0.001$ ) compared with etanercept, after 6 months' treatment.

Conclusions: In this population, infliximab was associated with a higher risk of requiring intensification of DMARD co-therapy than the other anti-TNF agents and a significant dose escalation over time. Analysis of RA disease activity indicated a reduced therapeutic response to infliximab after the first 6 months of treatment, suggestive of acquired drug resistance.
$\mathrm{N}$ ew disease modifying antirheumatic drugs (DMARDs) have become available since 1999. Biological agents, such as anti-tumour necrosis factor $\alpha$ (anti-TNF), dramatically improve the signs and symptoms of rheumatoid arthritis (RA) refractory to conventional treatment. ${ }^{1-3}$ The chemical structure, pharmacokinetic properties, and specific mechanisms of TNF inhibition of available anti-TNF agents differ: infliximab (INF) (Remicade; Centocor INC, Malvern, PA, USA) is a chimeric monoclonal anti-TNF antibody (human IgGK/mouse Fv); adalimumab (ADL) (Humira; Abbott Laboratories, Illinois, USA) is a fully human monoclonal anti-TNF antibody; whereas etanercept (ETN) (Enbrel; Amgen, Inc, Thousand Oaks, CA, USA) is an engineered TNF receptor (humanised protein) acting as a competitive inhibitor of $\mathrm{TNF} \alpha$ and $\beta$. The unique pharmacological properties of these agents have been associated with different rates of opportunistic granulomatous infections ${ }^{45}$ and are thought to explain why some anti-TNF agents work in some chronic inflammatory conditions and not in others. ${ }^{6}{ }^{7}$ It is not known whether these differences affect their long term therapeutic effectiveness or the potential development of drug resistance in RA.

In clinical practice, loss of effectiveness of long term DMARD treatment is a common problem. ${ }^{8}$ Acquired drug resistance or gradual drug failure has been described with most traditional DMARDs ${ }^{8-11}$ and is also starting to be recognised with anti-TNF agents. ${ }^{12}{ }^{13}$ Not all patients with
RA respond to the standard dosage of anti-TNF agents ${ }^{14} ; 28-$ $58 \%$ of all patients with RA show little response to these drugs in large randomised trials. ${ }^{15}$ Acquired resistance to DMARD treatment in RA has been measured by analysing use of additional DMARD co-therapy, ${ }^{16}$ anti-TNF dose escalation, ${ }^{11}$ and drug discontinuation rates ("drug survival" $^{\prime \prime}{ }^{8}{ }^{10}{ }^{16-18}$ These outcomes reflect the common therapeutic options a physician has when faced with loss of DMARD effectiveness ${ }^{19}$ : for patients not fully responsive to anti-TNF agents, physicians may increase co-therapy with traditional DMARDs, increase the anti-TNF dose, or decide to stop the current anti-TNF treatment and switch to other treatments. Dosage escalation has been observed with infliximab, ${ }^{19-25}$ but this may not a be valid measure of drug resistance for antiTNF agents without a flexible dosing regimen (ETN, ADL), in which case intensification of traditional DMARD Co-therapy and drug discontinuation might be more adequate outcomes.

This study aimed at investigating acquired drug resistance to anti-TNF treatments in a population based observational cohort of patients with RA. We examined intensification of DMARD co-therapy, progressive dose escalation, and drug

\footnotetext{
Abbreviations: $A D L$, adalimumab; $A N O V A$, analysis of variance; $\mathrm{Cl}$ confidence interval; DAS28, 28 joint count Disease Activity Score; DMARDs, disease modifying antirheumatic drugs; ETN, etanercept; $H A Q$, Health Assessment Questionnaire; HR, hazard ratio; INF, infliximab; RA, rheumatoid arthritis; RF, rheumatoid factor; SCQM, Swiss Clinical Quality Management; TNF, tumour necrosis factor
} 
discontinuation rates of the three available anti-TNF agents. In addition, we explored underlying pathways leading to these therapeutic adjustments in relationship to RA disease activity.

\section{PATIENTS AND METHODS \\ Study population}

Regulatory agencies in Switzerland have requested continuous monitoring of all patients receiving costly biological agents. ${ }^{26}$ The Swiss Clinical Quality Management of RA (SCQM) system ${ }^{27}$ was established by the Swiss Society of Rheumatology and elected to follow up all patients with RA starting to receive anti-TNF agents. The patient's rheumatologist or primary care physician are incited to enrol their patient in the SCQM by allowing them to deduct the costs of anti-TNF drugs from their global treatment expenditure scrutinised by the health authorities; this contributes to a high enrolment rate. Based on a comparison with sales data from the industry, between 70 and $80 \%$ of all Swiss patients with RA receiving anti-TNF agents are included in the SCQM. Patients are enrolled at the start of anti-TNF therapy and followed up prospectively. The SCQM includes measurements of disease activity, radiographic damage, adverse drug reactions, and RA symptoms. ${ }^{26}{ }^{27}$ Clinical information is collected systematically by the patient's physician every 612 months and further updated at every significant change in antirheumatic treatment. The accuracy of medication data provided by the physicians-including start and stop dateswas confirmed against records from the pharmaceutical industry and participants' self reported information. Patients come from a wide variety of clinical settings: $40 \%$ private rheumatology practices, 30\% non-academic centres, and 30\% academic centres. This analysis includes data collected between January 1998 and the end of September 2004. The inclusion criteria for this analysis were a diagnosis of RA by a rheumatologist and treatment with INF, ETN, or ADL.

\section{Outcomes}

We considered three outcomes that operationally define drug resistance ${ }^{16}$ : increase in concomitant DMARD treatment, dosage escalation in anti-TNF agents, and interruption of the current anti-TNF therapy. Traditional DMARD co-therapy was defined as concomitant prescription of methotrexate, leflunomide, sulfasalazine, ciclosporin, hydroxychloroquine, or azathioprine with an anti-TNF agent. Increase in concomitant DMARD treatment was defined by a rise in the weekly dose of traditional DMARDs or by the introduction of a new DMARD in addition to the current treatment. Replacement of one concomitant DMARD by another was not considered an increase in DMARD co-therapy. Dose escalation can occur either by increasing the dose of an anti-TNF agent or by shortening the dispensation interval. To take into account both of these possibilities, we computed each patient's average weekly dose of anti-TNF therapy $(\mathrm{mg} /$ week) at each time point. We examined drug retention of current anti-TNF therapies using the time until drug discontinuation, independently of the reason that led to drug interruption. Drug discontinuation rates or "drug survival rates" reflect both the patients' and doctors' satisfaction with a given treatment and thus represent a summary measure of the overall treatment effectiveness and tolerability. ${ }^{17} 18$

To link the therapeutic changes in anti-TNF therapy and/or in DMARD co-therapy with possible loss of drug effectiveness, we examined RA disease activity in a secondary analysis using the 28 joint count Disease Activity Score (DAS28). ${ }^{28}$ The DAS28 is a validated physician assessment of disease activity in RA, which includes the number of swollen joints, the number of tender joints, and the erythrocyte sedimentation rate. $^{28}$ The DAS28 ranges from 0 to 10 , where 10 represents maximum disease activity. Other important covariates such as the RA Disease Activity Index (RADAI), ${ }^{29}$ rheumatoid factor (RF) positivity, the Stanford Health Assessment Questionnaire (HAQ), ${ }^{30}$ previous failure of anti-TNF agents, and disease duration-defined as the time between symptom onset and enrolment-were also extracted from the SCQM database.

\section{Analysis}

Baseline disease characteristics of the three anti-TNF agents were compared. The significance of differences in mean values of continuous variables was assessed with one way analysis of variance (ANOVA) for normally distributed variables and with the Kruskal-Wallis test for non-normally distributed variables. For dichotomous variables, Pearson's $\chi^{2}$ test was used. All statistical tests were two sided and evaluated at the 0.05 significance level. To account for multiple pairwise comparisons, Bonferroni's procedure was used to adjust $\mathrm{p}$ values and confidence intervals (CIs). The statistical analysis was performed with Stata version 8.2 for Windows (Stata Statistical Software, Texas, USA).

The time to intensification of DMARD co-therapy and the time to discontinuation of anti-TNF agents ("drug survival") were analysed with Cox proportional hazards models. ${ }^{31}$ Survival curves of the time to intensification of DMARD Cotherapy and the time to discontinuation of anti-TNF agents ("drug survival") were produced using the Kaplan-Meier product-limit method. ${ }^{31}$ Dose escalation of anti-TNF agents and evolution of disease activity were analysed using generalised mixed models for longitudinal data. ${ }^{32}$ Rheumatoid factor positivity, baseline disease activity (DAS28), baseline functional disability (HAQ), disease duration, and failure of treatment with a previous anti-TNF agent were all considered confounders a priori and forced into the models. We tested other covariates using a backward stepwise selection approach. Additional covariates were included in the model only if found to be significant predictors or substantial confounders, using the $10 \%$ change in estimate criteria. $^{33}$ We also explored potential effect modification by co-therapy with traditional DMARDs and reported subgroup analyses when significant effect modification was present. For the final estimates, a robust estimator of the variance was used. All patients receiving anti-TNF agents were included in the analysis, though patients without follow up data only contributed information to baseline. Because incomplete follow up was generally due to recent initiation of anti-TNF therapy, we assumed absent follow up data to be missing at random.

\section{RESULTS}

A total of 1198 patients receiving anti-TNF treatment met the inclusion criteria, with assessments every 4 months on average. Some differences in baseline characteristics between the three groups were noted (table 1). In particular, for those receiving ADL another anti-TNF agent had failed in a higher proportion $(p<0.001)$, they had slightly lower disease activity, and less functional disability (HAQ) at baseline.

\section{Intensification of DMARD co-therapy}

The rate of intensification of traditional DMARD co-therapy was significantly different between the three treatments (ANOVA, $\mathrm{p}=0.006$ ) (fig 1 ). The hazard ratio (HR) of increasing traditional DMARD co-therapy over time with INF was 1.73 (99\% CI 1.19 to 2.51$)$ compared with the other anti-TNF agents, while there was no significant difference between ADL and ETN (Bonferroni, $\mathrm{p}=0.82$ ). The difference in DMARD co-therapy intensification between anti-TNF agents started to become statistically different only after 
Table 1 Baseline characteristics of patients at initiation of anti-TNF therapy

\begin{tabular}{|c|c|c|c|c|}
\hline Disease characteristics* & $\begin{array}{l}\text { Adalimumab (ADL) } \\
(n=317)\end{array}$ & $\begin{array}{l}\text { Infliximab (INF) } \\
(\mathrm{n}=362)\end{array}$ & $\begin{array}{l}\text { Etanercept (ETN) } \\
(n=519)\end{array}$ & p Value† \\
\hline Anti-TNF dose, median (IQR) & $40 \mathrm{mg} / 2 \mathrm{wk}(40-40)$ & $3.2 \mathrm{mg} / \mathrm{kg} \times 8 \mathrm{wk}(3.0-3.75)$ & $50 \mathrm{mg} / \mathrm{wk}(50-50)$ & \\
\hline Female (\%) & 74 & 75 & 74 & 0.89 \\
\hline Age (years) & $53.0(51.4$ to 54.7$)$ & $53.1(51.7$ to 54.5$)$ & $54.4(53.2$ to 55.6$)$ & 0.24 \\
\hline Disease duration (years), median (IQR) & $10.1(5.6-17.5)$ & $10.2(5.0-16.5)$ & $10.3(5.7-15.9)$ & 0.97 \\
\hline Rheumatoid factor+ (\%) & 82 & 81 & 78 & 0.33 \\
\hline Follow up (mo)‡, median (IQR) & $10.7(5.8-12.3)$ & $18.8(11.5-28.3)$ & $23.7(12.6-35.8)$ & $<0.001$ \\
\hline Previous failure of anti-TNF agent (\%) & 39 & 12 & 7 & $<0.001$ \\
\hline Disease activity score (DAS28) & $4.19(4.02$ to 4.36$)$ & $4.54(4.38$ to 4.7$)$ & $4.72(4.59$ to 4.85$)$ & $<0.001$ \\
\hline RADAl & $4.02(3.6$ to 4.5$)$ & $4.59(4.37$ to 4.81$)$ & 4.85 (4.67 to 5.03 ) & $<0.001$ \\
\hline Function disability (HAQ) & $1.25(1.18$ to 1.33$)$ & $1.37(1.29$ to 1.44$)$ & $1.37(1.31$ to 1.43$)$ & 0.04 \\
\hline Concomitant DMARD use (\%) & 53 & 93 & 64 & \\
\hline Methotrexate (\%) & 37 & 70 & 40 & \\
\hline Leflunomide $(\%)$ & 10 & 10 & 10 & \\
\hline Sulfasalazine (\%) & 4 & 6 & 6 & \\
\hline Other DMARD (\%) & 2 & 7 & 8 & \\
\hline Glucocorticoid use (\%) & 41 & 56 & 60 & $<0.001$ \\
\hline
\end{tabular}

*Values are given as means with $95 \%$ confidence intervals $(\mathrm{Cl})$ if not otherwise indicated. When not normally distributed variables, medians and interquartile ranges (IQRs) are reported; †one way analysis of variance (ANOVA) of means or medians for continuous variables. $\chi^{2}$ Test for dichotomous variables; $\ddagger$ for patients with follow up data only, respectively $n=107, n=295, n=425$.

DAS28, Disease Activity Score based on 28 joints; RADAl, Rheumatoid Arthritis Disease Activity Index; HAQ, Health Assessment Questionnaire; DMARD classical disease modifying antirheumatic drug; rheumatoid factor+, proportion of rheumatoid factor positive patients.

1.2 years of treatment. Significant predictors of DMARD intensification were previous failure of another anti-TNF agent $(\mathrm{HR}=2.14,95 \%$ CI 1.42 to 3.23$)$, RF positivity $(\mathrm{HR}=1.64,95 \%$ CI 1.07 to 2.51$)$, and disease duration ( $\mathrm{HR}=0.98,95 \%$ CI 0.96 to 0.99 ). As expected, starting INF without concomitant DMARDs significantly increased the need for subsequent intensification of co-therapy ( $\mathrm{HR}=4.5 \mathrm{l}$, 95\% CI 2.64 to 7.71 ), but this was not the case with ETN or ADL. No difference was observed between anti-TNF agents in glucocorticoid intensification or glucocorticoid reduction. Various sensitivity analyses with different definitions of DMARD co-therapy intensification, accounting for simultaneous changes in glucocorticoid dose, provided very similar results.

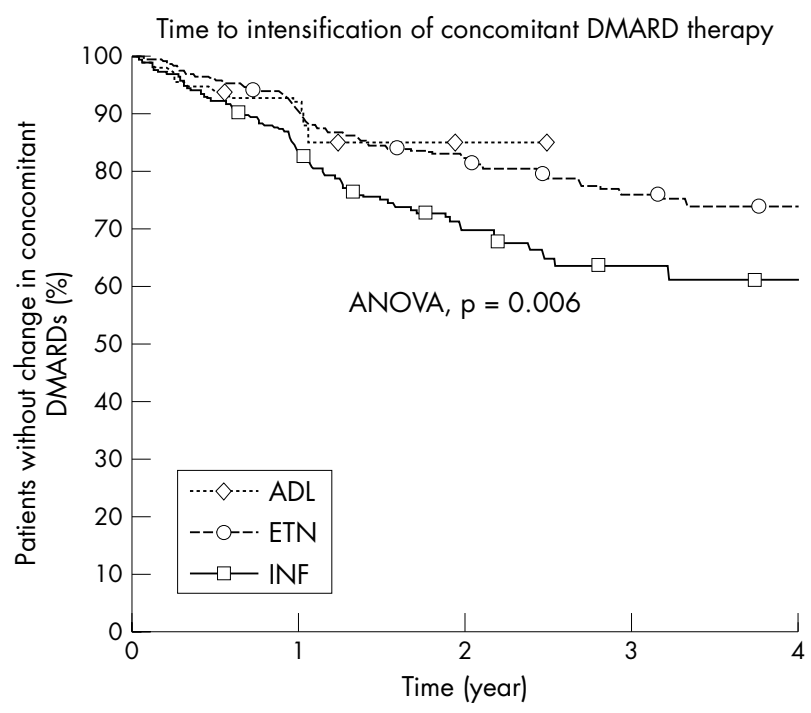

Figure 1 The Kaplan-Meier curve for time to intensification of concomitant traditional DMARD treatment after anti-TNF initiation (dosage increase of an existing DMARD or initiation of an additional DMARD). The survivor curve was adjusted for RF positivity, baseline disease activity scores (DAS28), level of functional disability at baseline (HAQ), and failure of previous anti-TNF agents. ADL, adalimumab; INF, infliximab; ETN, etanercept.

\section{Dose escalation}

Dose escalation was also significantly different between the three anti-TNF agents (ANOVA, $\mathrm{p}<0.001$ ), with only INF demonstrating a significant change in dosage over time (fig 2). The average dose escalation was $3.01 \mathrm{mg} /$ week (99\% CI 2.0 to 4.03 ) after the first year of INF treatment, which represents an average increase of $24 \mathrm{mg}$ per 2 month period or $+12 \%$ in INF dose. Furthermore, the average INF dose continued to escalate significantly after the first year of treatment and reached $4.60 \mathrm{mg} /$ week (99\% CI 2.84 to 6.36 ), which represents an average increase of $37 \mathrm{mg}$ per 2 month period or $+18 \%$ in INF dose. Dose escalation of INF was significantly higher in patients without concomitant DMARD treatment $(5.5 \mathrm{mg} /$ week at 1 year, $99 \%$ CI 1.9 to 9.01 ). No significant change in dose from baseline was apparent with ETN (Bonferroni, $\mathrm{p}=0.08$ ) or with ADL (Bonferroni,

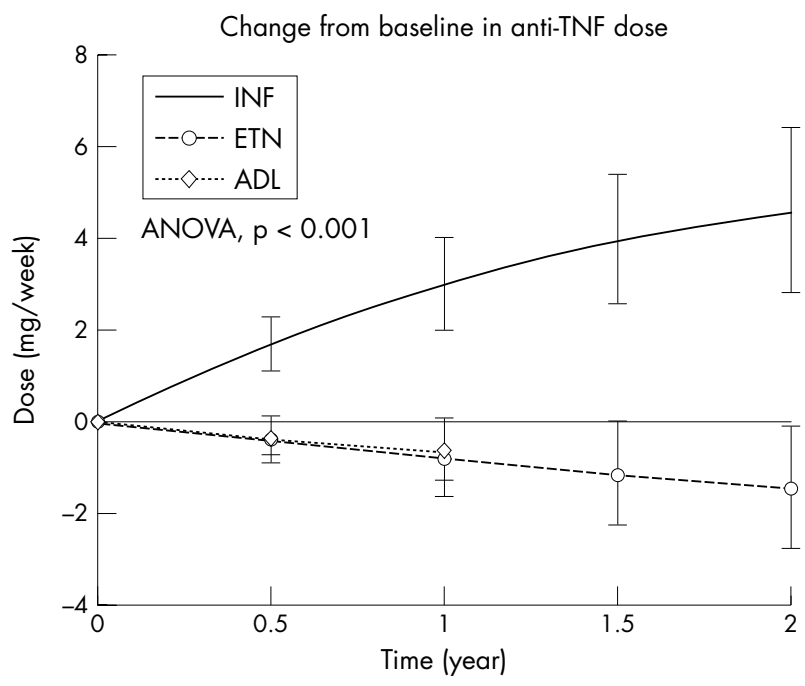

Figure 2 Anti-TNF dose is represented in $\mathrm{mg} /$ week for all anti-TNF agents to allow comparison. The vertical lines represent the $99 \%$ confidence interval of the mean. The evolution of the mean dose is adjusted for the proportion of RF positivity, baseline disease activity (DAS28), baseline levels of functional disability (HAQ), age, and failure of treatment with a previous anti-TNF agent. The evolution of the ADL dose is not represented after 1 year because data were scarce. INF, infliximab; ETN, etanercept; ADL, adalimumab. 


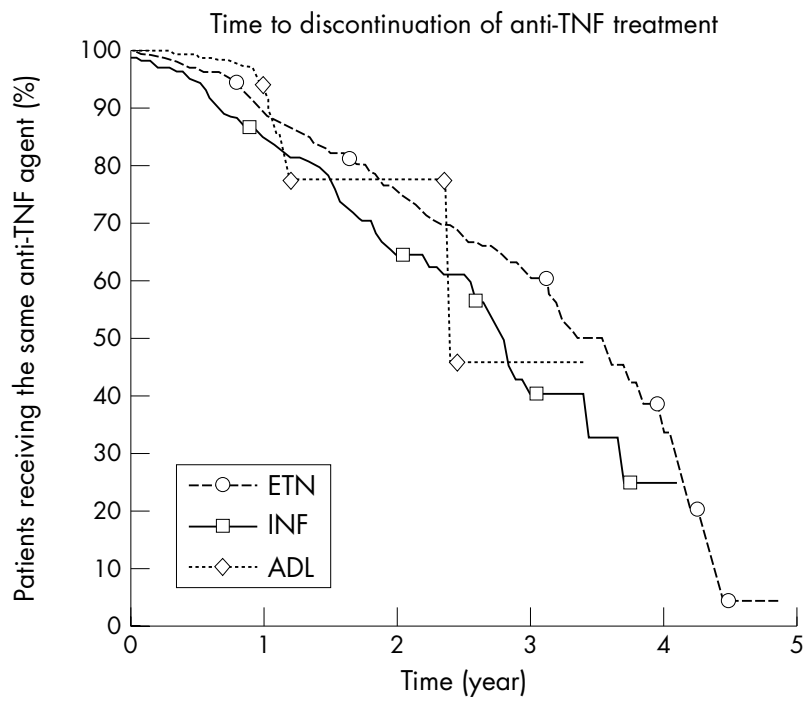

Figure 3 The Kaplan-Meier curve for time to discontinuation of antiTNF agents ("drug survival"). The survivor curve was adjusted for RF positivity, baseline disease activity scores (DAS28), level of functional disability at baseline (HAQ), year of initiation, and failure of previous anti-TNF agents. ADL, adalimumab; INF, infliximab; ETN, etanercept.

$\mathrm{p}=0.06$ ). However, progressive dose escalation may not be a valid measure of drug resistance for anti-TNF agents not commonly used with a flexible dosing regimen (ETN, ADL). Of the other covariates, only RF positivity significantly influenced changes in dose $(p=0.025)$.

\section{Discontinuation of treatment}

The discontinuation rates were not significantly different between the treatments (ANOVA, p =0.67) (fig 3). A total of 1450.5 patient-years of anti-TNF therapy and 300 cases of treatment interruption were examined. The overall discontinuation rate of anti-TNF agents was relatively low with a median drug survival of 3.21 years $(\mathrm{IQR}=1.43-4.52)$. The HR of discontinuing treatment was 1.11 (99\% CI 0.89 to 1.40 ) for INF compared with ETN and ADL, 0.97 (99\% CI 0.71 to 1.15) for ADL compared with INF and ETN, and 0.91 (99\% CI 0.71 to 1.15 ) for ETN compared with INF and ADL. Significant predictors of discontinuation were increased disability at baseline as measured by the HAQ ( $\mathrm{HR}=1.22$, $95 \%$ CI 1.00 to 1.48 ) and calendar year of treatment initiation $(\mathrm{HR}=2.01,95 \% \mathrm{CI} 1.71$ to 2.36$)$, which reflects the increasing proportion of patients starting biological agents after previous anti-TNF agents have failed and a greater availability of therapeutic alternatives favouring treatment switches over time. As expected, previous failure of another anti-TNF agent increased the rate of drug discontinuation by $77 \%$ ( $\mathrm{HR}=1.77,95 \%$ CI 1.23 to 2.54$)$ in a crude analysis. In a subgroup analysis, we explored the effect of DMARD cotherapy on anti-TNF discontinuation: in patients starting anti-TNF agents with concomitant DMARDs, discontinuation rates were significantly lower with ETN ( $\mathrm{HR}=0.66,99 \% \mathrm{CI}$ 0.45 to 0.98 ) than with INF or ADL.

\section{Therapeutic effectiveness on RA disease activity}

The evolution of RA disease activity (DAS28) over time differed significantly between anti-TNF agents (ANOVA $\mathrm{p}<0.001$ ) (fig 4). During the first 6 months of treatment, rates of improvement in disease activity varied little between the three groups, but thereafter the curves separated: with INF the DAS28 worsened by 0.14 (99\% CI 0.01 to 0.27 ) during the following year, compared with a non-significant improvement of 0.13 (99\% CI 0.03 to 0.27 ) with ETN during

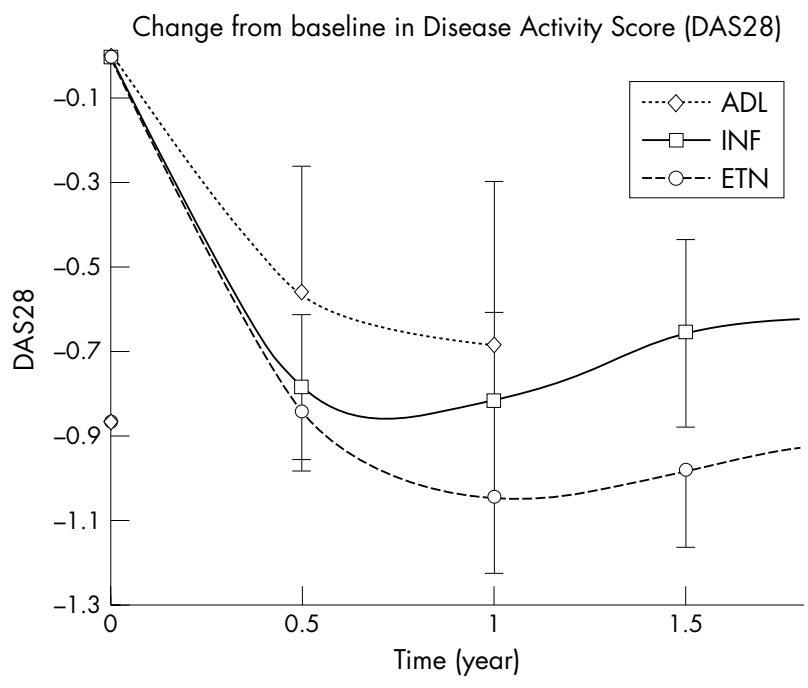

Figure 4 The evolution after initiation of anti-TNF agents of two measures of RA disease activity is displayed. Figure 4 represents the change from baseline in the Disease Activity Score (DAS28); range 0 to 10 , where 10 represents maximum disease activity. The vertical bars represent the $99 \%$ confidence interval for the mean. Results are adjusted for RF positivity, baseline disease activity score (DAS28), level of functional disability at baseline (HAQ), and failure of previous anti-TNF agents. ADL, adalimumab; INF, infliximab; ETN, etanercept.

the same period. Data for ADL were insufficient beyond 1 year of treatment to allow reliable comparisons. After the first year of treatment, the DAS28 had improved by 0.68 ( $99 \%$ CI 0.30 to 1.07) with ADL, by 0.82 (99\% CI 0.61 to 1.03 ) with INF, and by 1.05 (99\% CI 0.87 to 1.23 ) with ETN. Depending on the absolute level of disease activity, DAS28 improvements between 0.6 and 1.2 are considered moderate therapeutic responses and improvements $>1.2$ are considered good therapeutic responses (EULAR response criteria). ${ }^{34}$ After the first year of treatment, $47 \%$ (99\% CI 35\% to 59\%) of patients were classified as having a moderate or good response to $\mathrm{ADL}, 53 \%$ (99\% CI $44 \%$ to $61 \%$ ) to INF, and $66 \%$ (99\% CI $59 \%$ to $72 \%)$ to ETN.

\section{DISCUSSION}

We studied operational features of drug resistance to antiTNF agents in RA by analysing intensification of DMARD cotherapy, gradual dose escalation, and drug discontinuation rates in a population based cohort of 1198 patients with RA receiving INF, ADL, or ETN. Intensification of traditional DMARD Co-therapy over time was significantly higher with INF than with the other anti-TNF agents and progressive dose escalation was evident for INF, but not for ETN or ADL. No significant difference in discontinuation rates was seen. Analyses of RA disease activity over time indicated a lower therapeutic response to INF after the first 6 months of treatment compared with the other anti-TNF agents. With INF, lower therapeutic responses on the DAS28 were significantly associated with dose escalation and higher risk of concomitant DMARD intensification. Overall, these data suggest a gradual decrease of the therapeutic effectiveness of INF or the development of drug resistance occurring after the first half-year of treatment.

The rate of intensification of DMARD co-therapy was significantly higher with INF than with ETN or ADL $(\mathrm{HR}=1.73(99 \%$ CI 1.19 to 2.51$))$. A recent randomised controlled trial has established that ETN with concomitant MTX is more efficacious than ETN alone, ${ }^{35}$ and observational studies have confirmed that anti-TNF agents with concomitant DMARD treatment are more effective in preventing 
progression of radiographic damage than anti-TNF agents alone. $^{36}$ Intensification of concomitant DMARD treatment can thus represent an alternative to dose escalation of antiTNF agents and be a proxy for acquired drug resistance, ${ }^{16}$ in particular for biological agents whose dose is not commonly modified (ETN, ADL).

Not all patients with RA respond to the suggested initial INF dose of $3 \mathrm{mg} / \mathrm{kg}$. Higher serum levels of INF in the ATTRACT trial were associated with increased clinical response, reduction in $\mathrm{C}$ reactive protein levels, and reduced radiographic joint damage progression, which suggested a dose-response relationship. ${ }^{14}$ Subsequently, a flexible dosing regimen has been accepted for INF in some countries, including Switzerland, which allows dose increases up to $10 \mathrm{mg} / \mathrm{kg}$ and reductions in the dosing interval to every 4 weeks. Gradual dose escalation of INF has since been observed in several RA cohorts. ${ }^{19-25}$ The dose increase in an American cohort was $36 \%$ at 1 year, ${ }^{21}$ significantly more than in our cohort $(12 \%)$, which might reflect a difference in clinical practice and in patient population. It is not clear whether this dose escalation reflects initial dose adjustments of INF therapy or acquired drug resistance to this agent. In this study and in others, ${ }^{21}$ dose escalation of INF was steepest during the first year of treatment, but continued to increase in the second year at a slower rate, suggesting that both phenomena may be involved. Because of the price of antiTNF therapy, these results have important economic and clinical implications. It has been estimated that INF therapy incurred a $25 \%$ increase in 1-year costs representing an average of $\$ 4200$ per patient per year in the USA. ${ }^{24} \mathrm{~A}$ higher INF dosage may also carry an increased risk of side effects. ${ }^{20} 37$

No significant difference in discontinuation rates was seen between the three anti-TNF agents $(p=0.67)$ after adjusting for confounders. This corroborates results of other studies on retention rates of anti-TNF agents. ${ }^{38-40}$ Drug discontinuation rates represent a useful summary measure of overall effectiveness of a treatment, even if the rate is influenced by the availability of therapeutic alternatives or the incidence of adverse drug reactions. Anti-TNF agents had relatively low discontinuation rates and median drug survival of 3.21 years $(\mathrm{IQR}=1.43-4.52)$ in this population. Severe adverse drug reactions were relatively infrequent in this cohort, ${ }^{41}$ suggesting that drug discontinuation denoted mostly unsatisfactory treatment response. ${ }^{21}$

In usual clinical practice, concomitant DMARD co-therapy or anti-TNF agents are increased only if disease activity is not satisfactorily controlled, and tapered only when disease activity is adequately reduced. To link the therapeutic adjustments with clinically important outcomes, we examined RA disease activity over time in the three treatment groups using an independent outcome measure. The average effect size of the DAS28 response to anti-TNF agents was moderate, with an overall improvement of 0.92 (95\% CI 0.81 to 1.03 ) after the first year. ${ }^{34}$ Similar values of DAS28 improvement have been observed in other cohorts: in Swedish patients with RA, INF treatment was associated with a median DAS28 improvement of 0.6 after 1 year. ${ }^{20}$ However, the course of disease activity differed significantly between INF and the other anti-TNF agents. After a similar initial improvement, RA disease activity tended to slow or increase with INF after the first 6 months of treatment compared with ETN. Smaller therapeutic responses on RA disease activity were associated with dose escalation and concomitant DMARD treatment intensification in INF treated patients. While the mechanisms of resistance to INF or other anti-TNF agents are still poorly understood, the presence of antibodies to INF has been shown to reduce clinical response to this drug in patients with Crohn's disease $^{1242}$ and is also thought to contribute to the loss of response over time in some patients with RA. Unique pharmacological properties of anti-TNF agents have been associated also with differences in the incidence of opportunistic granulomatous infections ${ }^{45}$ or the efficacy profiles in other chronic inflammatory conditions. ${ }^{67}$ Further research is needed to understand the mechanisms leading to loss of drug effectiveness with anti-TNF agents.

Some potential limitations inherent to the analysis of observational data need discussion. Firstly, in this study, there was no control over the treatment assignment. Because no rationale exists for considering that one anti-TNF agent is more efficacious than another, ${ }^{7}$ major confounding by indication between these agents is unlikely. However, ADL had a higher proportion of patients for whom another antiTNF agent had failed, slightly lower disease activity, and less functional disability (HAQ) at baseline. These differences probably reflect the fact that ADL is the latest of the three anti-TNF agents to come on the market and that indication for these drugs has widened with time. While we were able to adjust our analysis for potential confounding by levels of disease activity, functional disability, RF positivity, previous failure of anti-TNF agents, or time trends, we could not exclude the possibility of confounding by unmeasured factors. For example, we could not adjust for concomitant rheumatic diseases or for self medication use such as analgesics. Although potential unmeasured confounding is always a concern, we have no evidence of a systematic bias, which would imply a differential prevalence of comorbidities or of co-medication use between anti-TNF agents. Intensification or reduction of concomitant glucocorticoid treatment was not significantly different between anti-TNF agents.

Secondly, missing data are another concern with observational studies. To deal with this, we checked the accuracy of medication data provided by the physicians-including start and stop dates-against records from the pharmaceutical industry and participants' self report. However, some residual non-differential misclassification is possible, which would tend to bias the results towards the null. ${ }^{43}$ We included all patients with RA receiving anti-TNF agents since 1999, but for some patients $(31 \%$, table 1$)$ no follow up data were available yet, allowing these patients to contribute only baseline information. The median follow up of patients receiving $\mathrm{ADL}$, in particular, was only 10.7 (IQR $=5.8-$ 12.3) months because it is the last agent to be introduced, which limited the ability to examine its potential drug resistance. By far, the most common reason for incomplete follow up was the recent initiation of anti-TNF therapy, with insufficient time for follow up measurements. Baseline disease characteristics of patients without complete follow up were similar to those included in the present analysis (data not shown), suggesting that the subjects in the analysis were a representative sample of the whole population.

The definition of acquired therapeutic resistance for antirheumatic treatment is less characterised than for antimicrobial treatment, for example. ${ }^{16}$ Acquired resistance to DMARD treatment has been measured by analysing the use of additional DMARD co-therapy, ${ }^{16}$ anti-TNF dose escalation, ${ }^{11}$ and drug discontinuation rates, ${ }^{8}{ }^{10}$ 16-18 $^{18}$ which are only indirect measures of this concept. Further research is needed to understand the mechanisms of drug resistance to DMARDs and define outcome measures of drug resistance in RA. Moreover, we could not adjust this study's longitudinal analyses for changes in time dependent confounders such as simultaneous variations in dose of glucocorticoid or DMARD co-therapy, because this would have introduced biases ${ }^{44} \mathrm{We}$ accounted for changes in dose of glucocorticoid and DMARD co-therapy by examining intensification or reduction of these 
treatments over time independently. Strengths of this analysis include a true population based cohort, as all patients with RA receiving anti-TNF agents are requested to be enrolled by the Swiss authorities, and a systematic prospective ascertainment of a wide variety of disease characteristics.

In this population, INF was associated with a higher risk of requiring intensification of DMARD co-therapy and a significant dose escalation compared with the other antiTNF agents. Analyses of the evolution of RA disease activity indicated a reduced therapeutic response to INF after the first 6 months of treatment, suggesting gradual drug resistance to this treatment. Further research is needed to study the mechanisms of drug resistance to INF and other anti-TNF agents.

\section{ACKNOWLEDGEMENTS}

Axel Finckh is supported by the Kirkland Scholars Fellowship, by the Centocor Health Outcomes in Rheumatic Diseases (CHORD) fellowship, by an unrestricted research grant from Wyeth, and by NIH grant P60 AR47782. Julia F Simard is supported by the Kirkland Scholars Fellowship and by NIH grant T32 AI07535.

We are grateful to the SCQM staff for data management and support and to participating physicians and patients who made this study possible, to Dr Liang and Dr Costenbader, BWH, Boston for critical input into the manuscript.

SCQM has received grants from the Swiss Health authorities (BAG), the Swiss Academy for Medical Sciences (SAMW), and private companies (Wyeth, Abbott, Essex, Aventis, Bristol-Mayers, Mepha, Merck, Novartis, and Roche).

This work was supported by the Centocor Health Outcomes in Rheumatic Diseases fellowship, the Kirkland Scholars Fellowship, an unrestricted research grant from Wyeth and by NIH grant P60 AR47782.

We thank specifically those rheumatologists who enrolled large numbers of these patients (10 or more): A-M Chamot, Morges; P Wiedersheim, St Gallen; T Lehmann, Bern; H Tinner, Weinfelden; C Zenklusen, Neuchatel; B Müller, Sarnen; T Cunningham, Geneva; JL Meier, Delémont; Th Langenegger, Unterägeri; G Thiebaud, Geneva; JB Suter, Bern; F Wicht, Solothurn; M Saxer, Basel; P Pancaldi, Muralto We also thank especially participating rheumatology clinics who registered large numbers of these cases (20 or more): rheumatology divisions of the University Hospital of Zurich; the University Hospital of Vaud; the University Hospital of Bern; Kantonsspital Aarau; Kantonsspital Luzern; Felix-Platter-Spital, Basel.

\section{Authors' affiliations}

A Finckh, Department of Medicine, Division of Rheumatology, Immunology and Allergy, Section of Clinical Sciences, Brigham and Women's Hospital, Harvard Medical School, Boston, USA

J F Simard, Department of Epidemiology, Harvard School of Public Health, USA

A Finckh, C Gabay, P-A Guerne, Division of Rheumatology, University of Geneva, Switzerland

\section{REFERENCES}

1 Bathon JM, Martin RW, Fleischmann RM, Tesser JR, Schiff MH, Keystone EC, et al. A comparison of etanercept and methotrexate in patients with early rheumatoid arthritis. N Engl J Med 2000;343:1586-93.

2 Weinblatt ME, Keystone EC, Furst DE, Moreland LW, Weisman MH, Birbara CA, et al. Adalimumab, a fully human anti-tumor necrosis factor alpha monoclonal antibody, for the treatment of rheumatoid arthritis in patients taking concomitant methotrexate: the ARMADA trial. Arthritis Rheum 2003;48:35-45.

3 Lipsky PE, van der Heijde DM, St Clair EW, Furst DE, Breedveld FC, Kalden JR, et al. Infliximab and methotrexate in the treatment of rheumatoid arthritis. Anti-Tumor Necrosis Factor Trial in Rheumatoid Arthritis with Concomitant Therapy Study Group. N Engl J Med 2000;343:1594-602

4 Wallis RS, Broder MS, Wong JY, Hanson ME, Beenhouwer DO Granulomatous infectious diseases associated with tumor necrosis factor antagonists. Clin Infect Dis 2004;38:1261-5.

5 Gardam MA, Keystone EC, Menzies R, Manners S, Skamene E, Long R, et al. Anti-tumour necrosis factor agents and tuberculosis risk: mechanisms of action and clinical management. Lancet Infect Dis 2003;3:148-55

6 Van den Brande JM, Braat $H$, van den Brink GR, Versteeg HH, Baver CA, Hoedemaeker I, et al. Infliximab but not etanercept induces apoptosis in lamina propria T-lymphocytes from patients with Crohn's disease. Gastroenterology 2003;124:1774-85.

7 Haraoui $\mathbf{B}$. Is there a rationale for switching from one anti-tumor necrosis factor agent to another? J Rheumatol 2004;31:1021-2.

8 Maetzel A, Wong A, Strand V, Tugwell P, Wells G, Bombardier C. Metaanalysis of treatment termination rates among rheumatoid arthritis patients receiving disease-modifying anti-rheumatic drugs. Rheumatology (Oxford) 2000;39:975-81.

9 van der Heijden J, de Jong MC, Dijkmans BA, Lems WF, Oerlemans R, Kathmann I, et al. Development of sulfasalazine resistance in human T cells induces expression of the multidrug resistance transporter ABCG2 (BCRP) and augmented production of TNFalpha. Ann Rheum Dis 2004;63:138-43.

10 Morgan C, Lunt $M$, Brightwell $H$, Bradburn P, Fallow W, Lay $M$, et al. Contribution of patient related differences to multidrug resistance in rheumatoid arthritis. Ann Rheum Dis 2003;62:15-19.

11 Lambert CM, Sandhu S, Lochhead A, Hurst NP, McRorie E, Dhillon V. Dose escalation of parenteral methotrexate in active rheumatoid arthritis that has been unresponsive to conventional doses of methotrexate: a randomized, controlled trial. Arthritis Rheum 2004;50:364-71.

12 Baert F, Noman M, Vermeire S, Van Assche G, D' Haens G, Carbonez A, et al. Influence of immunogenicity on the long-term efficacy of infliximab in Crohn's disease. N Engl J Med 2003;348:601-8.

13 Buch MH, Conaghan PG, Quinn MA, Bingham SJ, Veale D, Emery P. True infliximab resistance in rheumatoid arthritis: a role for lymphotoxin alpha? Ann Rheum Dis 2004;63:1344-6.

14 St Clair EW, Wagner CL, Fasanmade AA, Wang B, Schaible T, Kavanaugh A, et al. The relationship of serum infliximab concentrations to clinical improvement in rheumatoid arthritis: results from ATTRACT, a multicenter, randomized, double-blind, placebo-controlled trial. Arthritis Rheum 2002;46: 1451-9.

15 Redlich K, Schett G, Steiner G, Hayer S, Wagner EF, Smolen JS. Rheumatoid arthritis therapy after tumor necrosis factor and interleukin-1 blockade. Arthritis Rheum 2003;48:3308-19.

16 Wolfe F, Michaud K, Stephenson B, Doyle J. Toward a definition and method of assessment of treatment failure and treatment effectiveness: the case of leflunomide versus methotrexate. J Rheumatol 2003:30:1725-32.

17 Wolfe F. The epidemiology of drug treatment failure in rheumatoid arthritis. Baillieres Clin Rheumatol 1995;9:619-32

18 Wolfe F, Hawley DJ, Cathey MA. Termination of slow acting antirheumatic therapy in rheumatoid arthritis: a 14-year prospective evaluation of 1017 consecutive starts. J Rheumatol 1990:17:994-1002.

19 Sidiropoulos P, Bertsias G, Kritikos HD, Kouroumali H, Voudouris K, Boumpas DT. Infliximab treatment for rheumatoid arthritis, with dose titration based on the Disease Activity Score: dose adjustments are common but not always sufficient to assure sustained benefit. Ann Rheum Dis 2004;63:144-8.

20 van Vollenhoven RF, Brannemark S, Klareskog L. Dose escalation of infliximab in clinical practice: improvements seen may be explained by a regression-like effect. Ann Rheum Dis 2004;63:426-30.

21 Stern R, Wolfe F. Infliximab dose and clinical status: results of 2 studies in 1642 patients with rheumatoid arthritis, J Rheumatol 2004:31:1538-45.

22 Louis M, Rauch J, Armstrong M, Fitzcharles MA. Induction of autoantibodies during prolonged treatment with infliximab. J Rheumatol 2003;30:2557-62.

23 Fitzcharles MA, Clayton D, Menard HA. The use of infliximab in academic rheumatology practice: an audit of early clinical experience. J Rheumatol 2002;29:2525-30.

24 Gilbert TD Jr, Smith D, Ollendorf DA. Patterns of use, dosing, and economic impact of biologic agent use in patients with rheumatoid arthritis: a retrospective cohort study. BMC Musculoskelet Disord 2004;5:36.

25 Geborek P, Crnkic M, Petersson IF, Saxne T. Etanercept, infliximab, and leflunomide in established rheumatoid arthritis: clinical experience using a structured follow up programme in southern Sweden. Ann Rheum Dis 2002;61:793-8.

26 Langenegger T, Fransen J, Forster A, Seitz M, Michel BA. [Clinical quality management in rheumatoid arthritis. ] Z Rheumatol, 2001;60:333-41.

27 Uitz E, Fransen J, Langenegger T, Stucki G. Clinical quality management in rheumatoid arthritis: putting theory into practice. Swiss clinical quality management in rheumatoid arthritis. Rheumatology (Oxford) 2000:39:542-9.

28 Prevoo ML, van't Hof MA, Kuper HH, van Leeuwen MA, van de Putte $L B$, van Riel PL. Modified disease activity scores that include twenty-eight-joint counts. Development and validation in a prospective longitudinal study of patients with rheumatoid arthritis. Arthritis Rheum 1995:38:44-8.

29 Stucki G, Liang MH, Stucki S, Bruhlmann P, Michel BA. A self-administered rheumatoid arthritis disease activity index (RADAI) for epidemiologic research. Psychometric properties and correlation with parameters of disease activity. Arthritis Rheum 1995;38:795-8.

30 Fries JF, Spitz P, Kraines RG, Holman HR. Measurement of patient outcome in arthritis. Arthritis Rheum 1980;23:137-45.

31 Rosner B. Hypothesis testing: person-time data. In: Duxbury, eds. Fundamentals of Biostatistics.5th ed. CA: Pacific Grove, 2000:677-736.

32 Skrondal A, Rabe-Hesketh S. Generalized latent variable modeling: multilevel, longitudinal and structural equation models. Boca Raton, $\mathrm{FL}$ Chapman \& Hall/CRC, 2004.

33 Mickey RM, Greenland S. The impact of confounder selection criteria on effect estimation. Am J Epidemiol 1989;129:125-37.

34 van Gestel AM, Prevoo ML, van't Hof MA, van Rijswijk MH, van de Putte $L B$, van Riel PL. Development and validation of the European League Against Rheumatism response criteria for rheumatoid arthritis. Comparison with the preliminary American College of Rheumatology and the World Health Organization/International League Against Rheumatism Criteria. Arthritis Rheum 1996;39:34-40 
35 Klareskog L, van der Heijde D, de Jager JP, Gough A, Kalden J, Malaise $M$, et al. Therapeutic effect of the combination of etanercept and methotrexate compared with each treatment alone in patients with rheumatoid arthritis: double-blind randomised controlled trial. Lancet 2004;363:675-81

36 Finckh A, Simard JF, Duryea J, Liang MH, Huang J, Daneel S, et al. The effectiveness of anti-tumor necrosis factor therapy in preventing progressive radiographic joint damage in rheumatoid arthritis: a population- based study. Arthritis Rheum 2006:54:54-9.

37 Neven N, Vis M, Voskuyl AE, Wolbink GJ, Nurmohamed MT, Dijkmans BA, et al. Adverse events in patients with rheumatoid arthritis treated with infliximab in daily clinical practice. Ann Rheum Dis 2005;64:645-6.

38 Flendrie $M$, Creemers MC, Welsing PM, van Riel PL. The influence of previous and concomitant leflunomide on the efficacy and safety of infliximab therapy in patients with rheumatoid arthritis; a longitudinal observational study. Rheumatology (Oxford) 2005;44:472-8.

39 Geborek P, Crnkic M, Petersson IF, Saxne T; South Swedish Arthritis Treatment Group. Etanercept, infliximab, and leflunomide in established rheumatoid arthritis: clinical experience using a structured follow up programme in southern Sweden. Ann Rheum Di 2002;61:793-8.

40 Zink A, Listing J, Kary S, Ramlau P, Stoyanova-Scholz M, von Hinueber U, et al. Treatment continuation in patients receiving biological agents or conventional DMARD therapy. Ann Rheum Dis 2005;64:1274-9.

41 Forster A, Lucia HG, Synove D, Dudler J, Stingelin S, Oertle S, et al. Serious infections and their predictors in a Swiss cohort of rheumatoid arthritis patients treated with TNF inhibitors. ACR 2003 Annual scientific meeting; 2003; Orlando. Arthritis Rheum 2003;48(suppl):S328.

42 Farrell RJ, Alsahli M, Jeen YT, Falchuk KR, Peppercorn MA, Michetti P. Intravenous hydrocortisone premedication reduces antibodies to infliximab in Crohn's disease: a randomized controlled trial. Gastroenterology 2003;124:917-24.

43 Rothman K, Greenland S. Precision and validity in epidemiologic studies. In: Modern epidemiology. Philadelphia: Lippincott Williams and Wilkins, 1998: 115-34.

44 Robins JM, Hernan MA, Brumback B. Marginal structural models and causal inference in epidemiology. Epidemiology 2000;11:550-60. 